\title{
Hyperchaos synchronization using univariate impulse control
}

\author{
Kun Tian, ${ }^{1}$ Chao Bai, ${ }^{2}$ Hai-Peng Ren $\odot,{ }^{1,2, *}$ and Celso Grebogi ${ }^{1,3}$ \\ ${ }^{1}$ Shaanxi Key Laboratory of Complex System Control and Intelligent Information Processing, Xi'an University of Technology, \\ Xi'an 710048, China \\ ${ }^{2}$ School of Mechatronics Engineering, Xi'an Technological University, Xi'an 710048, China \\ ${ }^{3}$ Institute for Complex System and Mathematical Biology, SUPA, University of Aberdeen AB24 3UE, United Kingdom
}

(Received 29 August 2019; published 25 November 2019)

\begin{abstract}
Rössler and Chen systems with time delay are shown to be hyperchaotic, which exhibits a more complex dynamics, including multiple positive Lyapunov exponents and infinite dimension. The hyperchaos has better application potential where hyperchaos synchronization is concerned. Univariate impulse control requires smaller perturbation to the response system, thus promising better performance. However, synchronization of two hyperchaotic systems using this control method is a challenging task due to the difficulty to guarantee synchronization stability using a minimum number of manipulated variables. In this paper, a univariate impulse control method is proposed for the synchronization of two hyperchaotic dynamics generated by time delay. A theorem is developed and proved to provide the sufficient conditions for the synchronization of time delay systems using the univariate impulse control. The upper bound of the impulse interval is proved to guarantee the asymptotic synchronization. Simulation and circuit experiment show the correctness of the analysis and the feasibility of the proposed method.
\end{abstract}

DOI: 10.1103/PhysRevE.100.052215

\section{INTRODUCTION}

Chaos [1-3], displaying sensitive dependence on initial conditions, has materialized in many fields. Chaos control [4] and synchronization [5] have lighted the way to apply chaos in the fields of engineering (secure communication [6-8] and vibration compactors [9]), physics (turbulence [10], plasma [11] and semiconductors lasers [12,13]), chemistry [14], ecology [15], and biology [16].

As a widespread physical phenomenon in nature, chaos synchronization has been investigated for nearly three decades. To achieve two systems' synchronization, various synchronization control methods have been proposed since the pioneering work of Pecora and Carroll [5], which includes adaptive control [17], robust control [18], and others [19]. There are many practical synchronization experiments, including circuits [20,21] and laser systems [12,22]. Most of the existing synchronization control methods need to use the state information of the drive system at all times, except the impulse control [23].

The impulse control, which is the topic of this work, is attractive because it permits stabilization and synchronization of chaotic systems by only demanding small intervals of the state variables and smaller influence on the response system, thus providing a better energy efficiency and higher information security [24]. In 1990, Yang presented a seminal impulse control method based on the impulse differential equation [25]. The uniform asymptotic stability of the impulse differential system was established in Ref. [26]. Chen et al. investigated the sufficient conditions to guarantee the global asymptotic

*Corresponding author: renhaipeng@ xaut.edu.cn stability for impulse control and synchronization based on the Lyapunov function and linear matrix inequality [27]; some valuable results are given in $[28,29]$. The impulse synchronization has been investigated in various systems, including continuous systems [30,31], hyperchaotic systems [23,32,33], discrete chaotic systems [34], fractional-order systems [35], and a delay neural network [36]. Among those works, the reported impulse control methods are concerned with regulation of all states [32], which is difficult to apply in systems with uncontrollable variables, such as the Rössler system that describes chemical reactions. The univariate impulse control is capable of dealing with such problem, thus extending the application potential in addressing practical problems. The contribution of this work is listed as the following. First, in the existing impulse synchronization method, all variables in the response system are manipulated simultaneously $[23,25-$ 31,33-36]. Furthermore, the univariate impulse synchronization is proposed for chaotic systems, both with time delay, which requires less manipulated variables and thus less resources to achieve synchronization. Second, the asymptotic univariate impulse synchronization stability is investigated rigorously, providing the conditions for selecting synchronization controller parameters. Third, an impulse synchronization chaotic circuit and time delay unit are designed in order to validate the simulations and the analytic results. Our work achieves the theoretical analysis of univariate impulse synchronization for hyperchaos generated by time delay and then the circuit experimental verification is realized.

The remainder of the paper is organized as follows. In Sec. II, the uniform asymptotic stability of univariate impulse control and the sufficient condition to achieve the univariate impulse synchronization are derived based on the stability theory of the impulse delay-differential equation. Section III 
presents the simulation results of synchronization of two hyperchaotic systems using the univariate impulse control and one hyperchaotic circuit synchronization experiment to show the feasibility and effectiveness of the proposed method and the correctness of the analysis. Section IV gives the conclusions.

\section{IMPULSE SYNCHRONIZATION HYPERCHAOS GENERATED BY TIME DELAY}

For impulse synchronization, the most important issue is the synchronization stability. In this section, based on some basic definitions and a lemma from the literature [25], reviewed in Sec. II A, we derive the stability conditions of the impulse synchronization in Sec. II B and prove a theorem to guarantee the univariate impulse synchronization stability, which lays the theoretical foundation for the ensuing simulation and experiment.

\section{A. Preliminary details of impulse delay-differential equations}

The delay-differential equation is described by

$$
\dot{\mathbf{x}}=\mathbf{f}_{1}(t, \mathbf{x})+\mathbf{f}_{2}(t, \mathbf{x}(t-\tau))
$$

where $\mathbf{x}(t) \in R^{n}$ is the state vector, $\mathbf{f}_{1}, \mathbf{f}_{2}: R_{+} \times S(\rho) \rightarrow R^{n}$ are continuous function vectors on $\left(t_{k-1}, t_{k}\right] \times S(\rho)$, $S(\rho)=\left\{x \in R^{n} \mid\|x\|<\rho\right\}$, and $\|\cdot\|$ represents the $R^{n}$ space Euclidean norm.

In the impulse delay-differential equations, an impulse is added at $t_{k}$, described by

$$
\mathbf{x}\left(t_{k}^{+}\right)=\mathbf{x}\left(t_{k}^{-}\right)+\mathbf{C}\left[\tilde{\mathbf{x}}\left(t_{k}^{-}\right)-\mathbf{x}\left(t_{k}^{-}\right)\right], t=t_{k},
$$

where $\mathbf{x}$ represents the vector of the drive system, $\tilde{\mathbf{x}}$ represents the state vector of the response system, and $\mathbf{C}$ represents the impulse control matrix.

The impulse delay-differential equation is then given by

$$
\begin{aligned}
\dot{\mathbf{x}} & =\mathbf{f}_{1}(t, \mathbf{x})+\mathbf{f}_{2}(t, \mathbf{x}(t-\tau)), & & t \neq t_{k} \\
\Delta \mathbf{x} & =\mathbf{x}\left(t_{k}^{+}\right)-\mathbf{x}\left(t_{k}^{-}\right)=\boldsymbol{I}_{\boldsymbol{k}}(\mathbf{x}), & & t=t_{k}, k \in N \\
\mathbf{x}\left(t_{0}^{+}\right) & =\phi, & &
\end{aligned}
$$

where $\boldsymbol{I}_{\boldsymbol{k}}=\mathbf{C e}\left(t_{k}^{-}\right): S(\rho) \rightarrow R^{n}$ represents the impulse control variable. $\boldsymbol{I}_{\boldsymbol{k}}$ is the "jump" state at the time $t_{k}$, the impulse time $t_{k}$ satisfies $0<t_{0}<t_{1}<t_{2}<\cdots$ and $\lim _{k \rightarrow \infty} t_{k}=\infty$, and $\mathbf{e}\left(t_{k}^{-}\right)=\tilde{\mathbf{x}}\left(t_{k}^{-}\right)-\mathbf{x}\left(t_{k}^{-}\right)$.

Assuming that for all $k, \mathbf{f}(t, \mathbf{0}) \equiv 0$ and $\boldsymbol{I}_{k}(0)=0$, Eq. (3) has a trivial solution. The following definitions and lemmas are introduced [33]:

Definition 1. Let $V_{0}=\left\{V: R_{+} \times R_{+}^{n} \rightarrow R_{+}\right\}, V \in V_{0}$, for $[t, x(t)] \in[n T,(n+1) T] \times R_{+}^{n}$, and system (3) can be described as $\dot{\mathbf{x}}(t)=\mathbf{f}_{1}(t, \mathbf{x})+\mathbf{f}_{2}(t, \mathbf{x}(t-\tau))$. Then the upper right derivative of the solution of system (3) is defined as $D^{+} V[t, \mathbf{x}(t)]=\lim _{h \rightarrow 0^{+}} \sup \frac{1}{h}\{V[t+h, \mathbf{x}(t)+$ $h f(t, \mathbf{x}(t))]-V(t, \mathbf{x}(t))\}$.

Definition 2. Assume that $r \in N, D \subset R$, and $F \subset R$, $P C(D, F)$ denotes a piecewise continuous function from $D$ to $F$, namely, if $\phi \in P C(D, F)$, when $t \in D, t \neq t_{k}, \phi$ is a continuous function, except $t=t_{k}, \phi$ is a discontinuous function, but left side continuous. Denote $P C^{r}(D, F)$ as the $r$-order piecewise global differentiable function from $D$ to $F$, namely, if $\phi \in P C^{r}(D, F)$, then $\phi: D \rightarrow F, \frac{d^{r} \phi}{d t^{r}} \in P C(D, F)$.
Definition 3. $K_{1}=\left\{g \in C\left(R_{+}, R_{+}\right) \mid g(0)=0, g(s)>0\right.$, $\forall s>0\}, \quad K_{3}=\left\{g \in C\left(R_{+}, R_{+}\right) \mid g(0)=0, g(s)>0, \forall s>0\right.$, and $g$ is a nondecreasing function in $s$.

Lemma 1. Assume that $a, b, c \in K_{1}, \quad g \in K_{3}$, $p \in P C\left(R_{+}, R_{+}\right), \quad$ and $\quad V:[-r, \infty) \times S(\rho) \rightarrow R_{+}$, where $V$ is continuous on $\left(-r, t_{0}\right] \times S(\rho)$ and $\left(t_{k-1}, t_{k}\right] \times S(\rho), \quad k=1,2, \ldots$, for each $x \in S(\rho)$, and $k=0,1,2, \ldots, \quad \lim _{(t, y) \rightarrow\left(t_{k}^{-}, x\right)} V(t, y)=V\left(t_{k}^{-}, x\right) \quad$ exists; $V$ is locally Lipschitz in $x$ and the following conditions hold:

(1) $b(|\mathbf{x}|) \leqslant V(t, \mathbf{x}) \leqslant a(|\mathbf{x}|),(t, x) \in[-r, \infty) \times S(\rho)$.

(2) $D^{+} V(t, \phi(0)) \leqslant p(t) c[V(t, \phi(0))]$, for all $t \neq t_{k}$ in $R_{+}$, and $\phi \in P C([-r, 0], S(\rho))$ whenever $V(t, \phi(0)) \geqslant$ $g[V(t+s, \phi(s))]$ for $s \in[-r, 0]$.

(3) $V\left(t_{k}, \phi(0)+I_{k}\right) \leqslant g\left[V\left(t_{k}^{-}, \phi(0)\right)\right]$ for all $\left(t_{k}, \phi\right) \in$ $R_{+} \times P C\left([-r, 0], S\left(\rho_{1}\right)\right)$ for $\phi\left(0^{-}\right)=\phi(0)$.

(4) $\delta=\sup _{k \in z}\left\{\tau_{k}-\tau_{k-1}\right\}<\infty$, where $\delta$ is the impulse interval,

$$
\begin{aligned}
& M_{1}=\sup _{t>0} \int_{t}^{t+\delta} p(s) d s<\infty, \\
& M_{2}=\inf _{q>0} \int_{g(q)}^{q} \frac{d s}{c(s)}>M_{1} .
\end{aligned}
$$

Then, the trivial solution of system (3) is uniformly asymptotically stable.

\section{B. Time delay system synchronization using univariate impulse control}

Consider a general delay-differential equation given by

$$
\dot{\mathbf{X}}=\mathbf{A X}+\mathbf{B X}(t-\tau)+\boldsymbol{\varphi}(\mathbf{X})
$$

where $\boldsymbol{A} \in R^{n \times n}$ is the state matrix, $\boldsymbol{B}$ is the time delay gain matrix, $\varphi(\mathbf{x})$ is the nonlinear function, and $\tau$ is the delay time.

Separate the states of Eq. (4) into controllable and uncontrollable state variables, as follows:

$$
\begin{aligned}
\dot{\mathbf{x}} & =\mathbf{A}_{1} \mathbf{x}+\mathbf{B}_{1} \mathbf{x}(t-\tau)+\boldsymbol{\varphi}_{1}(\mathbf{x}, y), \\
\dot{y} & =A_{2} y+\varphi_{2}(\mathbf{x}, y),
\end{aligned}
$$

where $\mathbf{x}$ are the state variables and $y$ is a controllable scalar state variable, which is univariately manipulated.

To achieve synchronization with the drive system (5), the response system using univariate impulse control is given by

$$
\begin{aligned}
\dot{\tilde{\mathbf{x}}} & =\mathbf{A}_{1} \tilde{\mathbf{x}}+\mathbf{B}_{1} \tilde{\mathbf{x}}(t-\tau)+\boldsymbol{\varphi}_{1}(\tilde{\mathbf{x}}, \tilde{y}), \\
\dot{\tilde{y}} & =A_{2} \tilde{y}+\varphi_{2}(\tilde{\mathbf{x}}, \tilde{y}), \quad \Delta \tilde{\mathbf{x}}=0, \\
\Delta \tilde{y} & =\tilde{y}\left(t_{k}^{+}\right)-\tilde{y}\left(t_{k}^{-}\right)=c\left(\tilde{y}\left(t_{k}^{-}\right)-y\left(t_{k}^{-}\right)\right),
\end{aligned}
$$

where $\tilde{\mathbf{x}}$ and $\tilde{y}$ represent the state variables of the response system, $\Delta \tilde{\mathbf{x}}$ and $\Delta \tilde{y}$ represent the impulse control force at time $t_{k}$, and $c$ is a constant representing the impulse control amplitude. The other variables are the same as in Eq. (5). From the drive system (5) and the response system (6), we obtain the synchronization error system given by

$$
\begin{aligned}
\dot{\mathbf{e}}_{\mathbf{x}} & =\mathbf{A}_{1} \mathbf{e}_{\mathbf{x}}+\mathbf{B}_{1} \mathbf{e}_{\mathbf{x}}(t-\tau)+\mathbf{f}_{3}\left(\mathbf{e}_{\mathbf{x}}, e_{y}, \mathbf{x}, y, \tilde{\mathbf{x}}, \tilde{y}\right), \\
\dot{e}_{y} & =A_{2} e_{y}+f_{4}\left(\mathbf{e}_{\mathbf{x}}, e_{y}, \mathbf{x}, y, \tilde{\mathbf{x}}, \tilde{y}\right), \\
\Delta \mathbf{e}_{\mathbf{x}} & =0, \quad \Delta e_{y}=c e_{y}\left(t_{k}^{-}\right),
\end{aligned}
$$


where $\mathbf{e}_{\mathbf{x}}(t)=\tilde{\mathbf{x}}(t)-\mathbf{x}(t), e_{y}(t)=\tilde{y}(t)-y(t), \mathbf{f}_{3}\left(\mathbf{e}_{\mathbf{x}}, e_{y}, \mathbf{x}, y\right.$, $\tilde{\mathbf{x}}, \tilde{y})=\varphi_{1}(\tilde{\mathbf{x}}, \tilde{y})-\varphi_{1}(\mathbf{x}, y), \quad$ and $\quad f_{4}\left(\mathbf{e}_{\mathbf{x}}, e_{y}, \mathbf{x}, y, \tilde{\mathbf{x}}, \tilde{y}\right)=$ $\varphi_{2}(\tilde{\mathbf{x}}, \tilde{y})-\varphi_{2}(\mathbf{x}, y)$.

As long as the error system (7) is asymptotically stable at the origin, the response system (6) synchronizes with the drive system (5). Generally speaking, the stability of the synchronization is investigated using the conditional or maximum Lyapunov exponent $[5,13]$. In this paper, we investigate it in a rigorous way by a uniform asymptotic stability theorem of the error system (7), given as follows.

Theorem 1. Considering system (7), if the following two conditions are satisfied:

(1) there exist constants $L_{1}$ and $L_{2}$ so that $\left\|\varphi_{1}(\mathbf{x}, y)\right\|^{2} \leqslant$ $L_{1}\|\mathbf{x}\|^{2},\left\|\varphi_{2}(\mathbf{x}, y)\right\|^{2} \leqslant L_{2}\|\mathbf{x}\|^{2}$,

(2) $\quad M=\max \left\{\lambda_{\max }\left(\mathbf{A}_{1}{ }^{T}+\mathbf{A}_{1}\right)+2+L_{1}+\frac{\left\|\mathbf{B}_{1}\right\|^{2}}{\lambda_{\max }\left(\mathbf{I}^{2}\right)}, 2 \mathbf{A}_{2}+\right.$ $\left.1+L_{2}\right\}, 0<\delta<-\frac{\ln (1+c)^{2}}{M}$,

where $\lambda_{\max }(\cdot)$ is the maximum eigenvalue of the matrix in brackets and $\delta$ is the impulse interval, then the error system (7) is uniformly asymptotically stable at origin.

The proof of Theorem 1 is given in Sec. 1 of the Appendix.

\section{SIMULATION AND CIRCUIT EXPERIMENT RESULTS}

\section{A. Synchronization of the Rössler system with time delay}

The Rössler system is given as follows [2]:

$$
\begin{aligned}
& \dot{x}=-y-z, \\
& \dot{y}=x+\beta y, \\
& \dot{z}=\beta+z(x-\gamma),
\end{aligned}
$$

where for $\beta=0.1, \gamma=1.5$, the Rössler system has a periodic orbit.

The Rössler system with linear time delay feedback can be given as follows:

$$
\begin{aligned}
& \dot{x}=-y-z+k(x(t-\tau)-x(t)), \\
& \dot{y}=x+\beta y, \\
& \dot{z}=\beta+z(x-\gamma),
\end{aligned}
$$

and when $k=10.5$ and $\tau=1 s$, the time delay Rössler system is chaotic. Consider the equilibrium $\left(X^{*}, Y^{*}, Z^{*}\right)$ of system (9). Let $X=x-X^{*}, Y=y-Y^{*}, Z=z-Z^{*}$, and $\mathbf{X}^{T}=(X, Y, Z)$; then the Rössler system with time delay is transformed into

$$
\begin{aligned}
\dot{X} & =-Y-Z+K(X(t-\tau)-X), \\
\dot{Y} & =X+\beta Y, \\
\dot{Z} & =X Z+X Z^{*}+Z\left(X^{*}-\beta\right) \cdot w
\end{aligned}
$$

The impulse control is incorporated to the state variable $Y$; then we rewrite Eq. (10) in the form of Eq. (5) as $A_{1}=$ $\left[\begin{array}{cc}-K & -1 \\ Z^{*} & X^{*}-\beta\end{array}\right], B_{1}=\left[\begin{array}{l}K \\ 0\end{array}\right], \varphi_{1}=\left[\begin{array}{l}-Y \\ X Z\end{array}\right], A_{2}=\beta, \varphi_{2}=X$.

The error system is the same as in Eq. (7), and $\left\|\varphi_{1}(\mathbf{x}(t), y)\right\|^{2} \leqslant L_{1}\left\|\mathbf{e}_{\mathbf{x}}\right\|^{2}=30\left\|\mathbf{e}_{\mathbf{x}}\right\|^{2}, \quad\left\|\varphi_{2}(\mathbf{x}(t), y)\right\|^{2} \leqslant$ $L_{2}\left\|\mathbf{e}_{y}\right\|^{2}=3\left\|\mathbf{e}_{y}\right\|^{2}$. Select $c=-1.8$. From condition (2) (a)

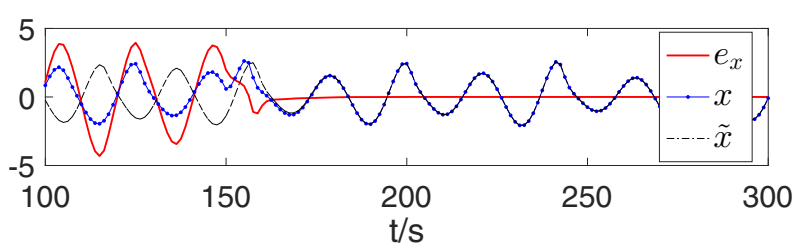

(b)

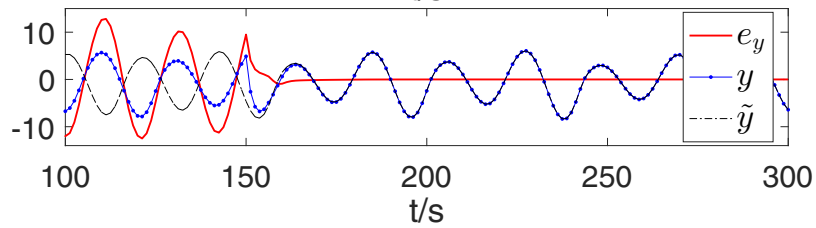

(c)

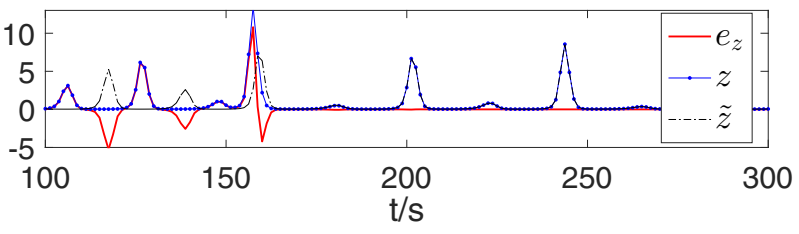

FIG. 1. The synchronization error curves (red solid line) $e_{x}, e_{y}$, $e_{z}$; the waveform of the state variable $x, y, z$ of the drive system (blue dotted solid line); and the waveform of the state variables $\tilde{x}, \tilde{y}, \tilde{z}$ of the response system (black dash-dotted line) of the Rössler system with time delay are presented in (a)-(c), respectively, where the impulse control is active from $t=150 \mathrm{~s}$.

in Theorem 1, we have the following:

(1) $M=\lambda_{\max }\left(\mathbf{A}_{1}^{T}+\mathbf{A}_{1}\right)+2+L_{1}+\frac{\left\|\mathbf{B}_{1}\right\|^{2}}{\lambda_{\max }\left(\mathbf{I}^{2}\right)}=63.5>0$,

(2) $0<\delta<-\frac{\ln (1+c)^{2}}{M}=-\frac{\ln (0.64)}{63.5}=0.0007$.

If we choose the impulse interval $\delta=0.005<0.007$, then all the conditions of Theorem 1 are satisfied. When the initial conditions of the drive system and the response system are $(-1,-0.2,-0.11)$ and $(1,0.2,0.1)$, respectively, the simulation results using univariate impulse control for $\delta=0.005$ are given in Fig. 1, where the impulse control is activated at $t=150 \mathrm{~s}$, and the waveform of $t=[0,99]$ is removed to avoid the initial condition effect. Figure 1(a) gives the curves of the drive system state $x$, response system state $\tilde{x}$ wave, and corresponding two state synchronization error $e_{x}$ as a blue dotted solid line, black dash-dotted line, and red solid line, respectively. Similarly, Figs. 1(b) and 1(c) give the curves of the corresponding variables of states $y$ and $z$, respectively. We learn from Fig. 1 that the synchronization is achieved when the impulse controller is active.

\section{B. Synchronization of the Chen systems with time delay}

The Chen system is given as follows [37]:

$$
\begin{aligned}
& \dot{x}(t)=a(y(t)-x(t)), \\
& \dot{y}(t)=(d-a) x(t)-x(t) z(t)+d y(t), \\
& \dot{z}(t)=x(t) y(t)-b z(t) .
\end{aligned}
$$

When $a=35, b=3, d=18.35978$, the Chen system is nonchaotic. The Chen system with linear time delay feedback is given by [38]

$$
\begin{aligned}
& \dot{x}(t)=a(y(t)-x(t)), \\
& \dot{y}(t)=(d-a) x(t)-x(t) z(t)+d y(t), \\
& \dot{z}(t)=x(t) y(t)-b z(t)+k(z(t)-z(t-\tau)),
\end{aligned}
$$


(a)

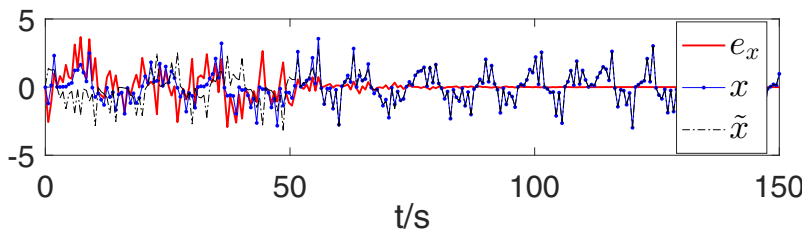

(b)

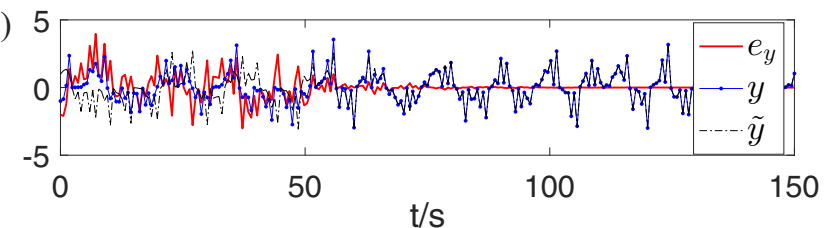

(c)

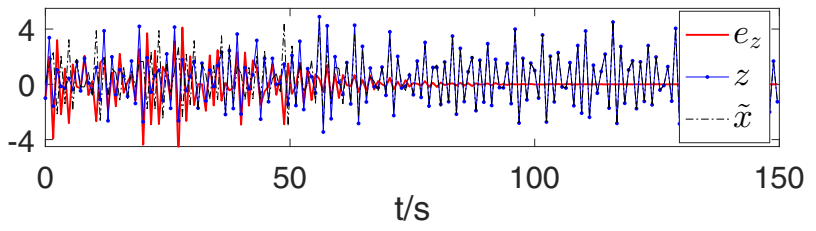

FIG. 2. The synchronization error curves $e_{x}, e_{y}, e_{z}$ (red solid line); the waveform of the state variables $x, y, z$ of the drive system (blue dotted solid line); and the waveform of the state variable $\tilde{x}, \tilde{y}, \tilde{z}$ of the response system (black dash-dotted line) are given in (a)-(c), respectively, where the impulse control is active from $t=50 \mathrm{~s}$.

and when $k=2.85$ and $\tau=0.3$, the initial value is $x(0)=2.27, \quad y(0)=2.27, \quad z(0)=1.72, \quad z(t)=0 \quad$ in $t \in[-0.3,0)$. The Chen system with time delay is hyperchaotic. The dynamical features of the chaotic system (12) are given in the Appendix.

We apply the impulse control to the state $\tilde{y}$ in the response system. The Chen system with linear time delay feedback control can be transformed into the form of Eq. (5), where $\mathbf{x}=\left[\begin{array}{c}x \\ z\end{array}\right], \boldsymbol{A}_{1}=\left[\begin{array}{cc}-a & 0 \\ 0 & -b+K^{\prime}\end{array}\right], \boldsymbol{B}_{1}=\left[\begin{array}{c}0 \\ -K\end{array}\right]$, $\boldsymbol{\varphi}_{1}(\mathbf{x}(t))=\left[\begin{array}{c}a y(t) \\ x(t) y(t)\end{array}\right], A_{2}=d, \varphi_{1}=(d-a) x(t)-x(t) z(t)$. The response system with impulse control is identical to the drive system, except for the impulse control.

The error system is given in the form of Eq. (7); $\boldsymbol{A}_{1}$ and $\boldsymbol{B}_{1}$ are the same as that in the drive system.

The impulse control gain is $c=-1.9$. $\left\|\varphi_{1}(\mathbf{x}(t), y)\right\|^{2} \leqslant$ $L_{1}\left\|\mathbf{e}_{\mathbf{x}}\right\|^{2}=16\left\|\mathbf{e}_{\mathbf{x}}\right\|^{2}, \quad\left\|\varphi_{2}(\mathbf{x}(t), y)\right\|^{2} \leqslant L_{2}\left\|\mathbf{e}_{y}\right\|^{2}=16\left\|\mathbf{e}_{y}\right\|^{2}$, and thus condition (1) in Theorem 1 is satisfied.

For condition (2) in Theorem 1, we have the following:

(1) $M=\lambda_{\max }\left(\mathbf{A}_{1}{ }^{T}+\mathbf{A}_{1}\right)+2+L_{1}+\frac{\left\|\mathbf{B}_{1}\right\|^{2}}{\lambda_{\max }\left(\mathbf{I}^{2}\right)}=91.8>0$,

(2) $0<\delta<-\frac{\ln (1+c)^{2}}{M}=-\frac{\ln (0.64)}{91.8}=0.0049$.

If we choose the impulse interval $\delta=0.0048<0.0049$, then all conditions of Theorem 1 are satisfied. The simulation results using impulse control for $\delta=0.0048$ are given in Fig. 2. Here the initial condition of the drive system and the response system are $(0.1,1,0.1)$ and $(-0.01,-1,-1)$, respectively, and the impulse control is activated at $t=50 \mathrm{~s}$. Figures 2(a)-2(c) are the first, second, and third states of the drive system, the response system, and the corresponding synchronization error curves, respectively. We see from Fig. 2 that the synchronization errors shown in the three subplots

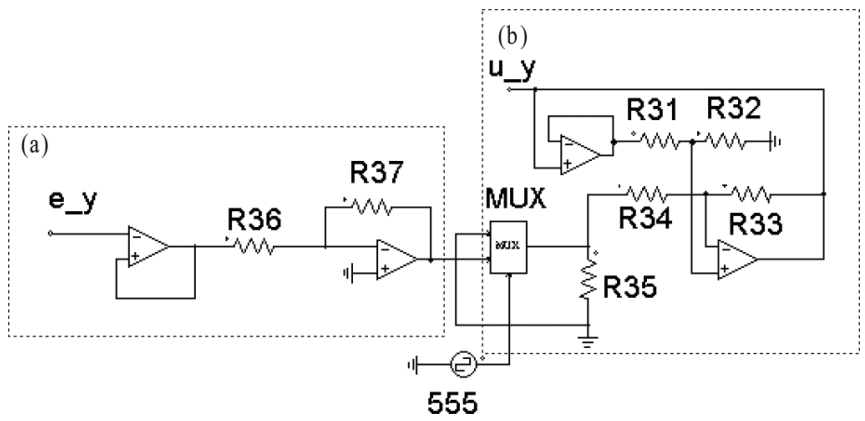

FIG. 3. The schematic diagram of the impulse control for the hyperchaotic Chen circuit synchronization.

with red solid lines tend to zero after the controller is activated at $t>50 \mathrm{~s}$. From Fig. 2, we learn that the synchronization is achieved using univariate impulse control.

\section{Chen circuit impulse synchronization experiment results}

The schematic diagram of impulse control circuit is given in Fig. 3, where block (a) is the proportional amplification. Error $e_{y}=\tilde{y}-y$ passes through the proportional amplifier, getting the proportional gain $c=-\frac{R_{37}}{R_{36}}$. The impulse interval is controlled by a 555 timer, which generates an impulse signal with $10 \%$ duty ratio and $0.0048 s$ period.

The multipath selector (CD4066) in Fig. 3 is used to select the inputs and to send the selected input to the output. When the impulse signal generated by the 555 timer is high, the control signal $u_{y}=c e_{y}$, otherwise $u_{y}=0$. Block (b) in Fig. 3 is used for incorporating the control into the state and power amplification.

The schematic diagram of the impulse synchronization of two circuits with one compressed in block (b) is given in Fig. 4, where block (b) is the drive system and block (a) represents the response system. The circuit parameters are summarized in Table I.

The experimental circuit photo is given in Fig. 5. The operation amplifiers used in the circuits are LF347N, the multipliers are AD633JN, the inverter is 74LS04, and the multichannel selector is CD4066. The experimental results are shown in Fig. 6.

Figures 6(a)-6(c) represent the states $x, y, z$ of the two systems and the corresponding synchronization errors [green line (channel 4) in each subplot], before impulse control is put into effect. Figures 6(d)-6(f) represent the states of the two systems and the corresponding synchronization errors after the controller is put into effect. From Fig. 6(a), we learn that the synchronization error between $x$ and $\tilde{x}$ oscillates between $-4 \mathrm{~V}$ and $+4 \mathrm{~V}$, before the controller takes over. However, in Fig. 6(d), the synchronization error $e_{x}$ is stable to zero after the controller is working, similarly for states $y$ and $z$ and their synchronization errors before and after the impulse control can be seen in other subplots, as described in the figure caption. Therefore, the synchronization is achieved after the univariate impulse control is put into effect in the circuits' experiment. 


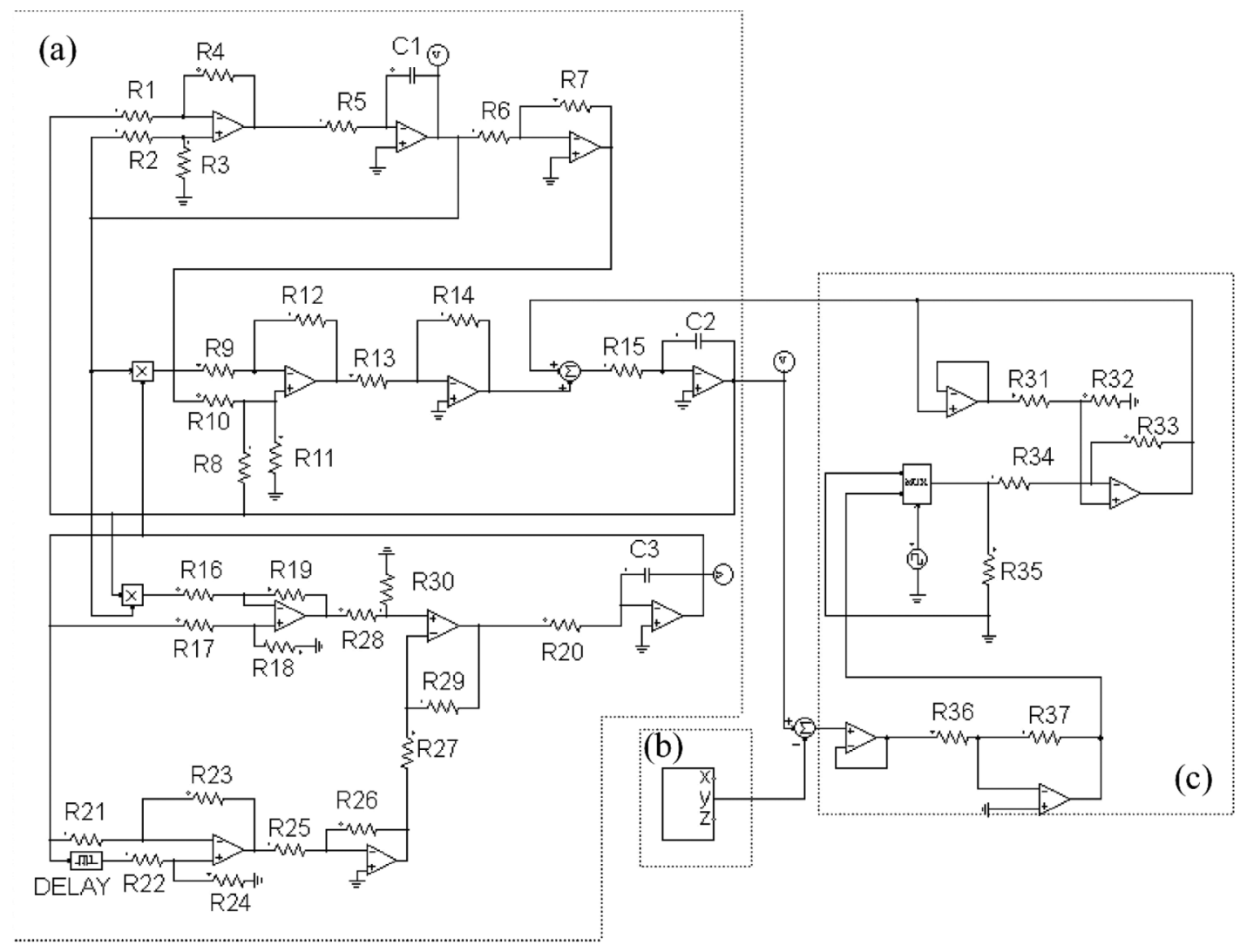

FIG. 4. The schematic diagram of the impulse control for circuits synchronization.

\section{CONCLUSION}

To conclude, on one hand, with the Lyapunov stability theory of the impulse delay-differential equation, we prove the sufficient condition for the univariate impulse synchronization with unidirectional coupling of two paradigmatic systems with time delay. On the other hand, we validate the correctness of the theory and effectiveness of the method, by simulations and an electrical circuit experiment.

TABLE I. Circuit component values.

\begin{tabular}{lc}
\hline $\mathrm{R} 1, \mathrm{R} 2, \mathrm{R} 3, \mathrm{R} 4, \mathrm{R} 6, \mathrm{R} 7, \mathrm{R} 13, \mathrm{R} 14, \mathrm{R} 16$, & \\
$\mathrm{R} 17, \mathrm{R} 19, \mathrm{R} 21, \mathrm{R} 22, \mathrm{R} 23, \mathrm{R} 24, \mathrm{R} 27, \mathrm{R} 28$ & $10 k \Omega$ \\
$\mathrm{R} 28, \mathrm{R} 29, \mathrm{R} 30$ & \\
$\mathrm{R} 5$ & $2.86 M \Omega$ \\
$\mathrm{R} 8$ & $16.5 k \Omega$ \\
$\mathrm{R} 9$ & $3 k \Omega$ \\
$\mathrm{R} 10$ & $18.5 k \Omega$ \\
R11 & $60 k \Omega$ \\
R12, R25, R31, R32, R33, R34, R36 & $1 k \Omega$ \\
R15 & $3.33 M \Omega$ \\
R18 & $1.765 k \Omega$ \\
R20 & $10 M \Omega$ \\
R26 & $285 \Omega$ \\
R35 & $12 \Omega$ \\
R37 & $1.8 k \Omega$ \\
$\mathrm{C} 1, \mathrm{C} 2, \mathrm{C} 3$ & $10000 P F$ \\
\hline
\end{tabular}

The advantage of the impulse control method is being intermittent, with less influence on the response system, and coupled with energy saving. It only employs control impulses to implement the synchronization. It is worth noticing that chaotic systems with time delay, combined with impulse control, can be used in secure communication, not only for having the high complexity of the secret key caused by the infinite-dimensional initial conditions needed for the delaydifferential equation [39], but also reducing the redundancy

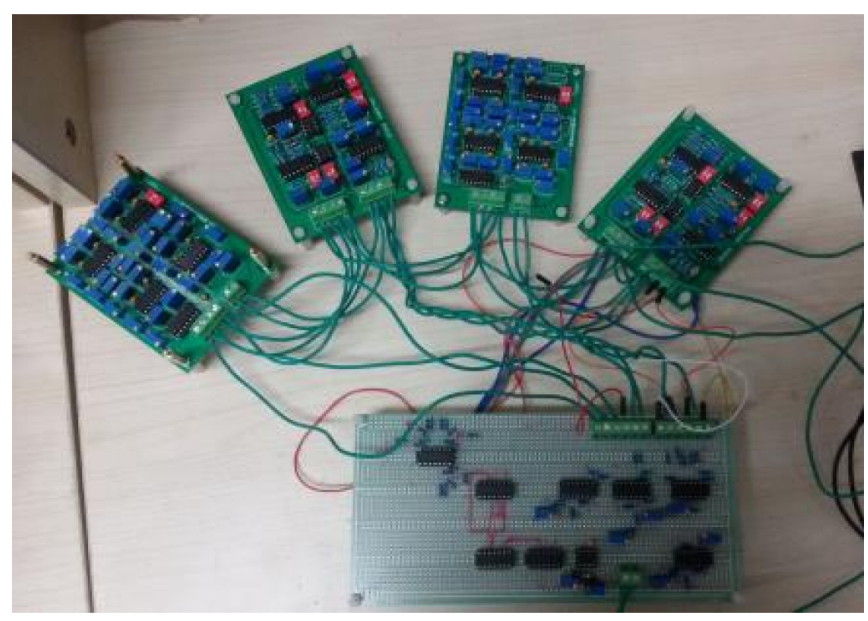

FIG. 5. The photo of the chaotic synchronization circuit. 


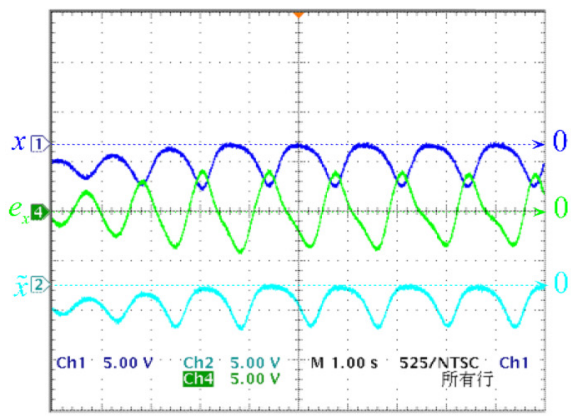

(a)

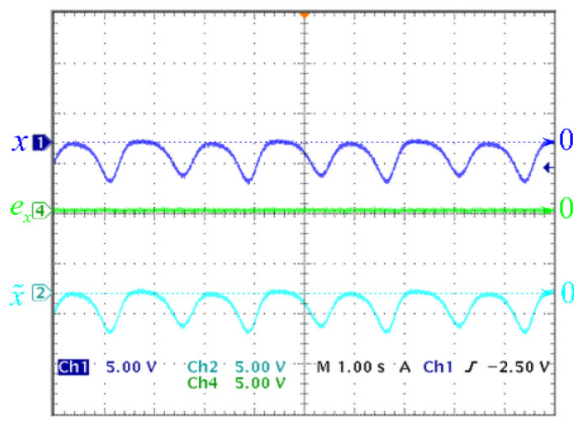

(d)

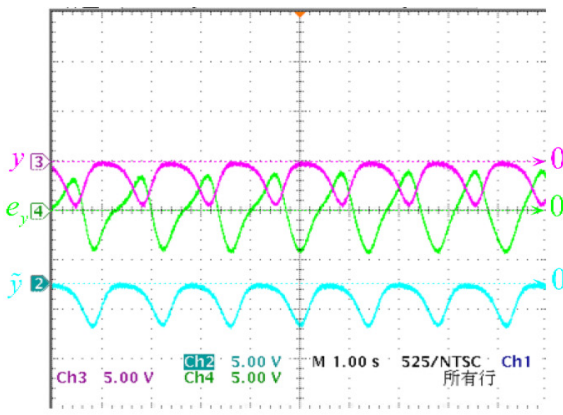

(b)

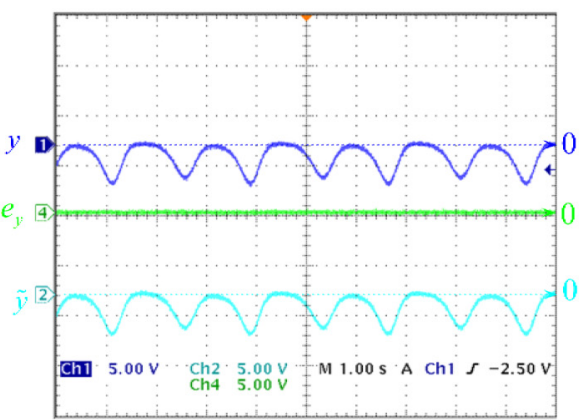

(e)

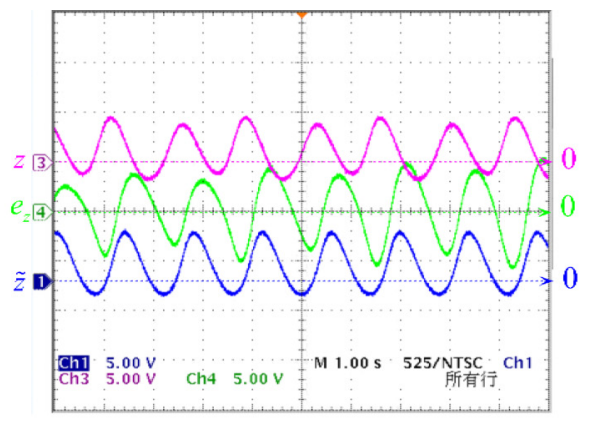

(c)

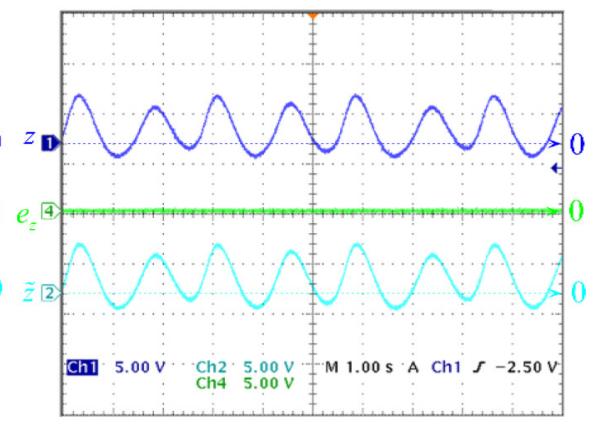

(f)

FIG. 6. The experimental waveforms: (a) drive system waveform $x$ (channel 1), response system waveform $\tilde{x}$ (channel 2), and error system waveform $e_{x}$ (channel 4) without impulse control; (b) drive system waveform $y$ (channel 3), response system waveform $\tilde{y}$ (channel 2), and error system waveform $e_{y}$ (channel 4) without impulse control; (c) drive system waveform $z$ (channel 3), response system waveform $\tilde{z}$ (channel 1 ), and error system waveform $e_{z}$ (channel 4) without impulse control; (d) drive system waveform $x$ (channel 1), response system waveform $\tilde{x}$ (channel 2), and error system waveform $e_{x}$ (channel 4) when impulse controller is active; (e) drive system waveform $y$ (channel 1), response system waveform $\tilde{y}$ (channel 2), and error system waveform $e_{y}$ (channel 4) when impulse controller is active; (f) drive system waveform $z$ (channel 1), response system waveform $\tilde{z}$ (channel 2), and error system waveform $e_{z}$ (channel 4) when impulse controller is active.

of the transmitting signal by which the required channel bandwidth can be effectively decreased.

The bidirectional coupled chaotic system's synchronization is a future possible extension of this work.

\section{ACKNOWLEDGMENTS}

The work is supported in part by the National Natural Science Foundation of China (Grant No. 61172070) and Shaanxi Provincial Special Support Program for Science and Technology Innovation Leader.

\section{APPENDIX}

\section{The proof of Theorem 1}

The proof of Theorem 1 is given as follows:

Proof. Select a Lyapunov function candidate as

$$
V(x)=\mathbf{e}^{T}(t) \mathbf{e}(t),
$$

where $\mathbf{e}(t)=\left[\begin{array}{ll}\mathbf{e}_{\mathbf{x}}(t) & e_{y}(t)\end{array}\right]^{T}$. For $t=t_{k}$,

$$
\begin{aligned}
\mathbf{V}\left(\mathbf{e}_{x}, e_{y}\right) & =\mathbf{e}_{x}^{T} \mathbf{e}_{x}+e_{y}^{T} e_{y} \\
& =\mathbf{e}_{x}^{T}\left(t_{k}^{-}\right) \mathbf{e}_{x}\left(t_{k}^{-}\right)+(1+c)^{2} e_{y}^{T}\left(t_{k}^{-}\right) e_{y}\left(t_{k}^{-}\right) \\
& \leqslant(1+c)^{2} \cdot \mathbf{V}\left(t_{k}^{-}, \mathbf{e}\left(t_{k}^{-}\right)\right) \\
& =g\left[\mathbf{V}\left(t_{k}^{-}, \mathbf{e}\left(t_{k}^{-}\right)\right)\right],
\end{aligned}
$$

where $g(\mathbf{V})=(1+c)^{2} \mathbf{V}$.
If $V(t, \mathbf{e}(t)) \geqslant g[V(t+s, \mathbf{e}(t+s))]$, for $-\tau \leqslant s \leqslant 0$, then $V(t, \mathbf{e}(t)) \geqslant(1+c)^{2} V(t+s, \mathbf{e}(t+s))$. Therefore,

$$
V(t, \mathbf{e}(t)) \geqslant(1+c)^{2}\|\mathbf{e}(t+s)\|^{2} .
$$

For $t \neq t_{k}$,

$$
\begin{aligned}
D^{+} V & (t, \mathbf{e}(t)) \\
= & \dot{\mathbf{e}}_{\mathbf{x}}^{T}(t) \mathbf{e}_{\mathbf{x}}(t)+\mathbf{e}_{\mathbf{x}}^{T}(t) \dot{\mathbf{e}}_{\mathbf{x}}(t)+\dot{\mathbf{e}}_{y}^{T}(t) \mathbf{e}_{y}(t)+\mathbf{e}_{y}^{T}(t) \dot{\mathbf{e}}_{y}(t) \\
= & {\left[\mathbf{A}_{1} e_{\mathbf{x}}(t)+\mathbf{B}_{1} e_{\mathbf{x}}(t-\tau)+\varphi_{1}(\mathbf{x}, y)\right]^{T} \mathbf{e}_{\mathbf{x}}(t) } \\
& +\mathbf{e}_{\mathbf{x}}^{T}(t)\left[\mathbf{A}_{1} \mathbf{e}_{\mathbf{x}}(t)+\mathbf{B}_{1} \mathbf{e}_{\mathbf{x}}(t-\tau)+\varphi_{1}(\mathbf{x}, y)\right] \\
& +2\left[\mathbf{A}_{2} \mathbf{e}_{y}(t)+\varphi_{2}(\mathbf{x}, y)\right]^{T} e_{y}(t) \\
\leqslant & \lambda_{\max }\left(\mathbf{A}_{1}^{T}+\mathbf{A}_{1}\right) \mathbf{e}_{\mathbf{x}}^{T}(t) \mathbf{e}_{\mathbf{x}}(t)+2 \mathbf{e}_{\mathbf{x}}^{T}(t) \mathbf{B}_{1} \mathbf{e}_{\mathbf{x}}(t-\tau) \\
& +2 \mathbf{e}_{\mathbf{x}}^{T}(t) \varphi_{1}(\mathbf{x}, y)+2 \mathbf{A}_{2} \mathbf{e}_{y}^{T}(t) \mathbf{e}_{y}(t)+2 \varphi_{2}(\mathbf{x}, y) \mathbf{e}_{y}(t) \\
\leqslant & \lambda_{\max }\left(\mathbf{A}_{1}^{T}+\mathbf{A}_{1}\right) \mathbf{e}_{\mathbf{x}}^{T}(t) \mathbf{e}_{\mathbf{x}}(t)+2\left\|\mathbf{e}_{\mathbf{x}}(t)\right\|^{2} \\
& +\left\|\mathbf{B}_{1}\right\|^{2}\left\|\mathbf{e}_{\mathbf{x}}(t-\tau)\right\|^{2}+\left\|\varphi_{1}(\mathbf{x}, y)\right\|^{2}+2 \mathbf{A}_{2} \mathbf{e}_{y}^{T}(t) \mathbf{e}_{y}(t) \\
& +\left\|\mathbf{e}_{y}(t)\right\|^{2}+\left\|\varphi_{2}(\mathbf{x}, y)\right\|^{2} \\
\leqslant & {\left[\lambda_{\max }\left(\mathbf{A}_{1}^{T}+\mathbf{A}_{1}\right)+2+L_{1}+\frac{\left\|\mathbf{B}_{1}\right\|^{2}}{\lambda_{\max }\left(\mathbf{I}^{2}\right)}\right] \mathbf{e}_{\mathbf{x}}^{T}(t) \mathbf{e}_{\mathbf{x}}(t) } \\
& +\left(2 \mathbf{A}_{2}+1+L_{2}\right) \mathbf{e}_{y}^{T}(t) \mathbf{e}_{y}(t) \\
\leqslant & p(t) V(t, \mathbf{e}(t)),
\end{aligned}
$$


where $\quad p(t)=\max \left\{\lambda_{\max }\left(\mathbf{A}_{1}{ }^{T}+\mathbf{A}_{1}\right)+2+L_{1}+\frac{\left\|\mathbf{B}_{1}\right\|^{2}}{\lambda_{\max }\left(\mathbf{I}^{2}\right)}\right.$, $\left.2 \mathbf{A}_{2}+1+L_{2}\right\}$, and assume $c(s)=s, M=p(t)$.

According to condition (4) of Lemma 1, we have

$$
\begin{aligned}
M_{2}-M_{1} & =\inf _{q>0} \int_{g(q)}^{q} \frac{d s}{c(s)}-\sup _{t>0} \int_{t}^{t+\delta} p(s) d s \\
& =\ln q-\ln g(q)-M \delta \\
& =-\ln g-M \delta>0 .
\end{aligned}
$$

According to condition (2) of Theorem 1, if the following conditions are held:

$$
\begin{aligned}
M= & \max \left\{\lambda_{\max }\left(\mathbf{A}_{1}{ }^{T}+\mathbf{A}_{1}\right)+2+L_{1}+\frac{\left\|\mathbf{B}_{1}\right\|^{2}}{\lambda_{\max }\left(\mathbf{I}^{2}\right)},\right. \\
& \left.2 \mathbf{A}_{2}+1+L_{2}\right\} \\
& 0<\delta<-\frac{\ln (1+c)^{2}}{M},
\end{aligned}
$$

then the error system (7) satisfies the conditions of Theorem 1 , and we have the conclusion that the error system is asymptotically stable.

\section{Time delay implementation using small time-lag circuits cascade}

Time delay can generate infinite-dimensional hyperchaos [40]. The Chen system with time delay is such an example, having multiple kinds of attractors, including the single scroll attractor [41], the double scroll attractors [38,40], and the composite multiscroll attractors [42], which possesses multiple positive Lyapunov exponents, more complex dynamics, and better application potential.

In order to produce the chaotic attractors in the experiment, we use a circuit to implement Eq. (12). The circuit is then divided into two parts: Chen circuit and delay circuit. Methods of delay circuit implementation include the delay-line based oscillators operating at very high and ultrahigh frequency ranges [43], a T-type LCL network [44], a digital sampling and replying with memory shift method $[38,45]$, the all-pass filter method [46], and chains of $n$ Bessel filters [47]. In this work, small time-lag units cascade are used to implement the needed time delay. Compared with the other methods, this method is simpler to implement [42]. The time-lag unit diagram is shown in Fig. 7. by

The transfer function of the small time-lag units is given

$$
G(s)=\frac{1}{1+T s}=\frac{1}{(1+R C s)},
$$

[1] E. N. Lorenz, J. Atmos. Sci. 20, 130 (1963).

[2] O. E. Rössler, Phys. Lett. A. 57, 397 (1976).

[3] M. Hénon, Commun. Math. Phys. 50, 69 (1976).

[4] E. Ott, C. Grebogi, and J. A. Yorke, Phys. Rev. Lett. 64, 1196 (1990).

[5] L. M. Pecora and T. L. Carroll, Phys. Rev. Lett. 64, 821 (1990).

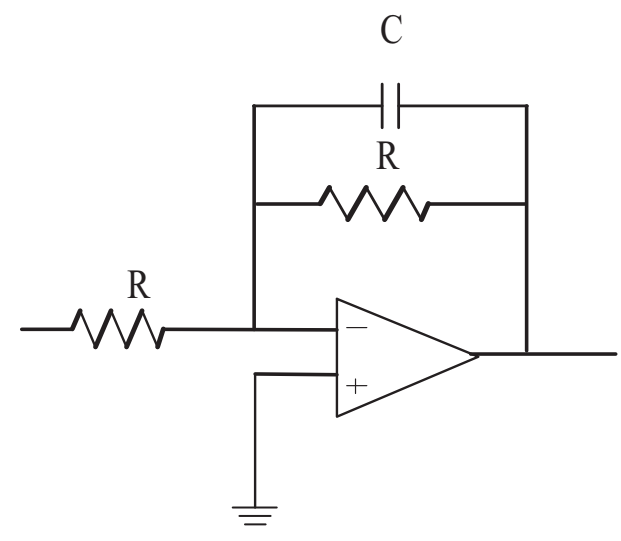

FIG. 7. The time-lag circuit unit.

where $T=R C=\frac{\tau}{n}$ is the time-lag constant. If $T$ is small enough, the phase frequency characteristics of the time-lag units are close to that of the delay unit. Amplitude and phase frequency properties of the $n$ units time-lag cascade are given as

$$
\left|G^{\prime}(j \omega)\right|=\left(\frac{K}{\sqrt{1+(T \omega)^{2}}}\right)^{n}, \angle G^{\prime}(j \omega)=-n * \arctan (T \omega) .
$$

If $n$ is large enough and $T$ is small enough, then the $n$ thorder time-lag units approximate the pure time delay unit [42]. This method of implementation of time delay has a modular structure and it is simple to implement. In order to make the cascading time-lag units have a unit gain as that of pure time delay, we need to add an amplitude compensation circuit.

\section{Lyapunov spectrum calculation of chaos with time delay}

Fifteen time-lag units are used to implement time delay. Then, an 18-order differential equation is derived to describe the behavior of the system with time delay, given as

$$
\begin{aligned}
& \dot{\mathbf{X}}=\mathbf{A X}+\mathbf{B}\left(\mathbf{X}-u_{n}\right)+\boldsymbol{\varphi}(\mathbf{X}), \\
& \dot{u}_{i}=\frac{1}{T}\left(u_{i-1}-u_{i}\right), i=1,2,3, \ldots, n,
\end{aligned}
$$

where $n=15$. The Lyapunov exponent spectrum is calculated by using the Jacobi matrix method. We can conclude that there are two positive Lyapunov exponents in both the Rössler system with time delay and in the Chen system with time delay. The positive Lyapunov exponents in the Rössler system with time delay are 0.24 and 0.22 . The positive Lyapunov exponents in the Chen system with time delay are 0.8055 and 0.12 . Both attractors are hyperchaotic attractors.
[6] H. P. Ren and C. Bai, Chinese Phys. B 24, 080503 (2015).

[7] B. Wang, S. M. Zhong, and X. C. Dong, Commun. Nonlinear Sci. Num. Simul. 39, 108 (2016).

[8] H. P. Ren, C. Bai, Z. Z. Huang, and C. Grebogi, Int. J. Bifurc. Chaos 27, 1750076 (2017). 
[9] H. P. Ren, American Patent application, No. PCT/CN2016/073418 (2016).

[10] T. Klinger, C. Schröder, D. Block, F. Greiner, and A. Piel, Phys. Plasmas 8, 1961 (2001).

[11] A. E. Hramov, A. A. Koronovskii, and I. S. Rempen, Chaos 16, 013123 (2006).

[12] M. C. Soriano, J. García-Ojalvo, C. R. Mirasso, and I. Fischer, Rev. Mod. Phys. 85, 421 (2013).

[13] S. Heiligenthal, T. Dahms, S. Yanchuk, T. Jüngling, V. Flunkert, I. Kanter, E. Schöll, and W. Kinzel, Phys. Rev. Lett. 107, 234102 (2011).

[14] T. V. O. Scheper, Chaos 27, 103122 (2017).

[15] D. Biswas, T. Banerjee, and J. Kurths, Chaos 27, 063110 (2017).

[16] L. A. Aguirre, L. L. Portes, and C. Letellier, Chaos 27, 103103 (2017).

[17] H. P. Ren and C. Z. Han, Chinese J. Sci. Instrum. 29, 792 (2008) (in Chinese).

[18] Y. Y. Hou, T. L. Liao, and J. J. Yan, Physica A 379, 81 (2007).

[19] S. Boccaletti, J. Kurths, G. Osipov, D. Valladares, and C. Zhou, Phys. Rep. 366, 1 (2002).

[20] P. Ruiz, J. M. Gutierrez, and J. Güemez, Chaos, Solitons Fractals 36, 635 (2008).

[21] U. E. Kocamaz, S. Cicek, and Y. Uyaroğlu, J. Circuit Syst. Comp. 27, 1850057 (2018).

[22] R. Roy and K. S. Thornburg, Jr., Phys. Rev. Lett. 72, 2009 (1994).

[23] C. D. Li, X. F. Liao, and X. Y. Zhang, Chaos 15, 023104 (2005).

[24] T. Yang and L. O. Chua, IEEE Trans. Circuits Syst. I, Fund. Theory Appl. 44, 976 (1997).

[25] T. Yang, IEEE T. Automat. Contr. 44, 1081 (1999).

[26] T. Yang, Impulsive Control Theory (Springer, Verlag, 2001).

[27] D. L. Chen, J. T. Sun, and C. S. Huang, Chaos Solitons Fractals 28, 213 (2006).
[28] R. Z. Luo, Phys. Lett. A 372, 648 (2008).

[29] C. Ma and X. Y. Wang, Nonlinear Dyn. 70, 551 (2012).

[30] J. T. Sun, Y. P. Zhang, and Q. D. Wu, Phys. Lett. A 298, 153 (2002).

[31] J. T. Sun and Y. P. Zhang, Phys. Lett. A 306, 306 (2003).

[32] H. P. Ren, K. Tian, and C. Bai, arXiv:1911.07021 (2019).

[33] X. Z. Liu and G. Ballinger, Comput. Math. Appl. 41, 903 (2001).

[34] L. P. Zhang, H. B. Jiang, and Q. S. Bi, Nonlinear Dyn. 59, 529 (2010).

[35] N. Liu, J. Fang, W. Deng, Z. J. Wu, and G. Q. Ding, Int. J. Control Autom. Syst. 16, 2839 (2018).

[36] W. He, F. Qian, and J. Cao, Neural Networks 85, 1 (2017).

[37] G. R. Chen and T. Ueta, Int. J. Bifurcation Chaos 9, 1465 (1999).

[38] H. P. Ren and W. C. Li, Commun. Nonlinear Sci. Num. Simul. 15, 3058 (2010).

[39] H. P. Ren, C. F. Zhao, and C. Grebogi, Int. J. Bifurcation Chaos (to be published).

[40] H. P. Ren, D. Liu, and C. Z. Han, Acta Phys. Sinica 55, 2694 (2006) (in Chinese).

[41] K. Tian, H. P. Ren, and C. Grebogi, Int. J. Bifurcation Chaos 29, 1950114 (2019).

[42] H. P. Ren, C. Bai, K. Tian, and C. Grebogi, Int. J. NonLin Mech. 94, 334 (2017).

[43] G. Mykolaitis, A. Tamaševičius, A. Čenys, and S. Bumeliene, Chao Soliton Fract. 17, 343 (2003).

[44] A. Namajūnas, K. Pyragas, and A. Tamaševičius, Phys. Lett. A. 201, 42 (1995).

[45] X. F. Wang, G. Q. Zhong, and K. S. Tang, IEEE. Trans. Circuit Syst. I 48, 1151 (2001).

[46] G. S. Hu, Int. J. Circ. Theor. App. 39, 79 (2009).

[47] A. Buscarino, L. Fortuna, M. Frasca, and G. Sciuto, IEEE. Trans. Circuit Syst. I 58, 1888 (2011). 